\title{
Monte Carlo simualtions of the hard square-triangle fluid close to melting
}

\author{
K. V. Tretiakov and K. W. Wojciechowski \\ Institute of Molecular Physics, Polish Academy of Sciences \\ Smoluchowskiego 17/19, 60-179 Poznań, Poland \\ kww@man.poznan.pl
}

\begin{abstract}
The system of hard squares and triangles of equal sides, for which the free volume approximation predicts entropic phase separation in the solid phase, is studied in the dense fluid region, close to melting. The standard Monte Carlo simulations are inconclusive concerning the possibility of the phase separation in the dense fluid. By applying correlated motions of the particles, we present a direct evidence that, in contrast to the dense solid phase, the squares and triangles mix in the fluid phase.
\end{abstract}

\section{Introduction}

The entropy is usually considered as one of main factors responsible for mixing of various substances both in the gas phase as well as in the condensed phases. Recently, however, it has been pointed out that the entropy can also stimulate the phase separation in systems composed of molecules which much differ in size [1] or in shape [2]. The phase separation in such systems is usually understood as the effect of the depletion forces [3], Recently, it has been noticed that at high densities, where the free volume approximation works well [4], the role of the driving force for spatial separation of various molecules can be attributed to the free volume entropy [5], Namely, when the free volume entropy per molecule (defined as the entropy of a molecule moving in an average cell formed by its (fixed) neighbours) reaches maximum, the average distance between the surface of a molecule and the surfaces of its neighbours (which form the cell) is smaller for large molecules than for small ones. Thus, the free volume entropy disfavours neighbouring pairs of molecules of different sizes. For molecules of different shapes the tendency to phase separation is also expected [5].

A simple model on which the theoretical predictions of Ref. [5] can be tested is a two-dimensional (2D) system of hard squares and triangles of equal sides, 
further referred to as the hard square-triangle model. At close packing, the hard square-triangle model was introduced by Collins [6] to study the nature of the melting transition in the hard disc system. Using the mean field approximation to this model Collins was able to reproduce, with a reasonable accuracy, both the solid and fluid branches of the hard disc system but was not able to obtain the phase transition between them. Kawamura, who incorporated into the calculations the short-range correlations of squares and triangles, succeeded to reproduce qualitatively the isotherm of the hard discs, including the melting transition [7]. He also noticed that the hard squres and triangles can form quasicrystalline structures at close packing and estimated the entropy of the quasicrystalline structures [8], These entropic quasicrystals were further studied by Widom [9] and Kalugin [10] who calculated their entropy exactly. Recently, the model has been studied by the free volume approximation in the vicinity of the close packing [5], These calculations suggest that triangles and squares should separate just below the close packing. Preliminary simulations performed by us support this conclusion. As the phase separation in this case is not caused by a trivial packing missfit of the particles (triangles and squares can tile the plane both being mixed or separated) this result encouraged us to check if the phenomenon of the entropic phase separation occures also in the fluid phase.

As it has been discussed in [5], for particles of different sizes and/or shapes, the free volume (FV) entropy (which corresponds to the vibrations of molecules in their cells formed by their neighbours) favours, in general, phase separation. The free volume entropy competes, however, with the degeneracy entropy which measures the number of essentially different distributions of the FV cells. The latter term is difficult to estimate if the microscopic structure of the system is not known. This is the case of the squares and triangles in the fluid phase. Thus, to check if the squares and triangles can separate there (in the fluid), we decided to perform Monte Carlo (MC) simulations of the system. Below we present preliminary results for the case when the packing fractions of both the molecules are nearly equal.

\section{MC simulations}

The role played by the free volume mechanism is larger at higher densities than at the lower ones [5], For this reason it is natural to search for possibility of a phase separation of the squares and triangles in the fluid at its highest density. As it can be seen in Fig. 1 and Fig.2, mixtures of the squares and the triangles of the concentration ratio close to one half melt spontaneously slightly above the

dimensionless pressure $\mathrm{p}^{*} \equiv \mathrm{p} \sigma^{2} / \mathrm{k}_{\mathrm{B}} \mathrm{T}=13$. Thus, it is reasonable to expect that the system at this pressure corresponds to a stable fluid phase. Most of the simulations described below were performed in the NpT ensemble at this pressure. 


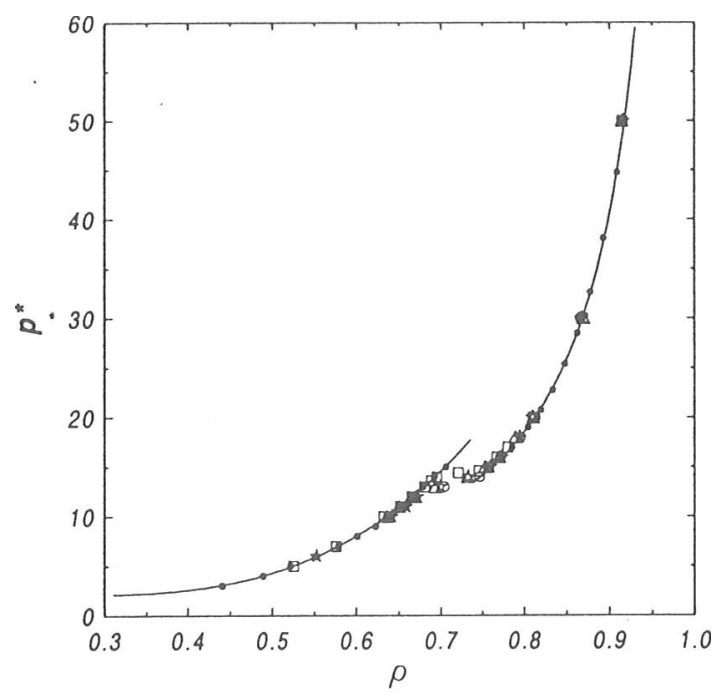

Fig.l Black dots represent the isotherm obtained by decreasing the pressure for the solid structure shown in Fig.3a. Open circles, pentagons, squares, and triangles represent the isotherms for the structures shown in Fig.2a-d. It can be seen that the spontaneous melting occurs in all these systems slightly above $\mathrm{p}^{*}=13$.
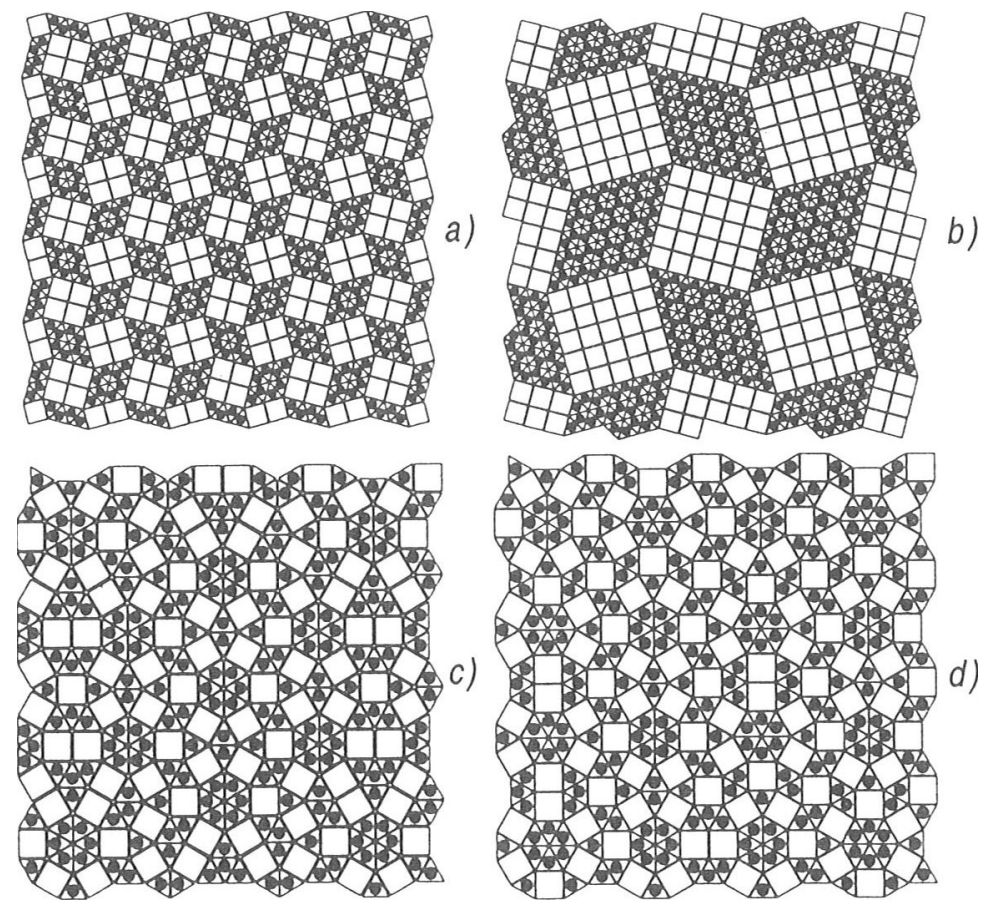

Fig.2 Some of the solid structures for which the expansion isotherms were computed. Centres of the triangles are indicated by black dots. 


\subsection{Standard Monte Carlo simulations}

We started with two experiments performed for extremely different initial configurations. The first simulation was initiated from the "mixed" crystalline phase shown in Fig.3a. The system constituted of $N_{s}=698$ hard squares and $N_{t}=1296$ hard triangles moving (via translational and rotational motions) in a periodic box of variable "volume" (area) and variable (rectangular) shape. The pressure was equal $\mathrm{p}^{*}=13$, and the initial relative volume, $\mathrm{v}^{*} \equiv 1 / \mathrm{p}^{*}=\mathrm{V} / \mathrm{V}_{\mathrm{cp}}=1.45$, was close to its equilibrium value, being slightly higher than that at which the solid spontaneously melted. $\mathrm{V}$ is the volume of the system, $\mathrm{V}_{\mathrm{cp}}$ corresponds to the volume of the close packed crystal, and $\rho^{*}$ is the relative density. Each MC cycle, further referred to as a standard MC cycle, consisted of a trial motion (including a random translation and rotation) performed for each molecule, i.e. of $N_{s}+N_{t}$ "molecular" motions, and one trial to change the volume and shape of the box. The acceptance ratio was kept close to 30 percent for the molecular motions and to 25 percent for the motions of the box of periodicity. The initial structure quickly melted, in about $10^{4}$ standard MC cycles. After that, formation of clusters of sizes slowly increasing with time was observed. This can be seen in snapshots of the system configurations shown in Fig.3b-d. This
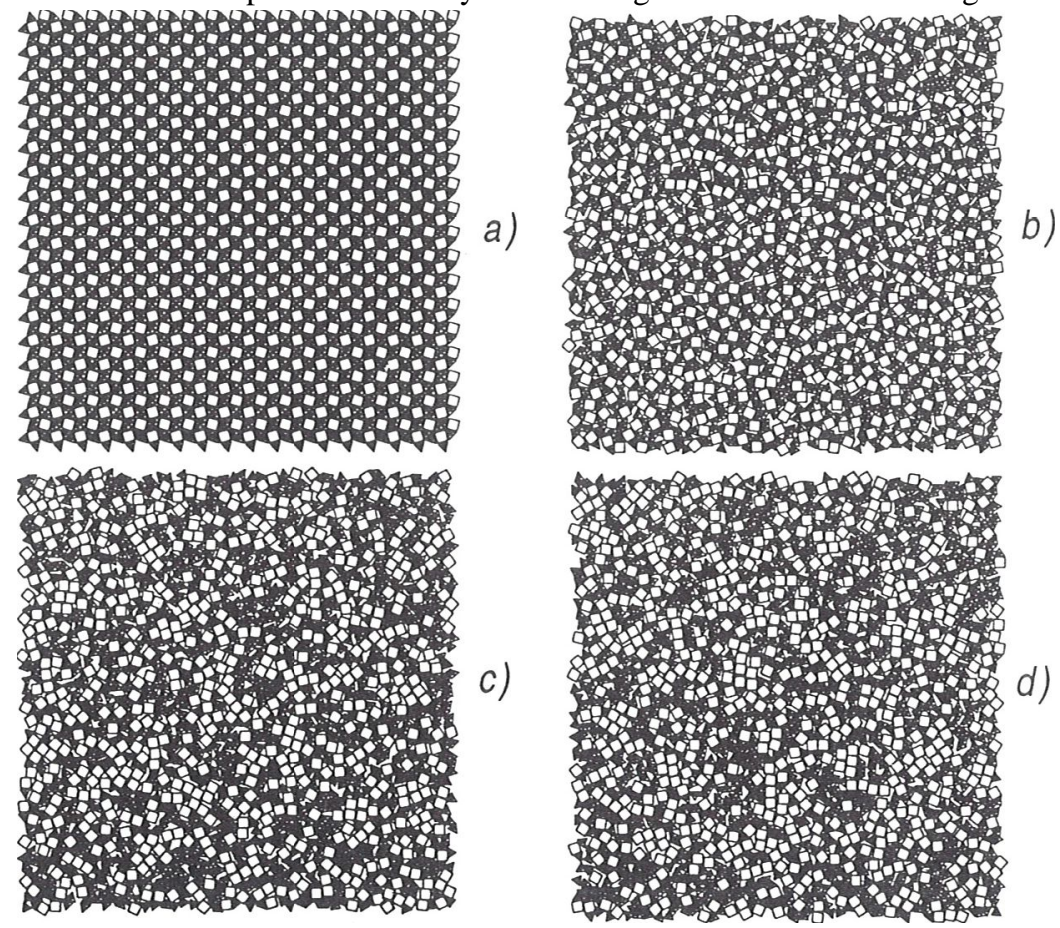

Fig.3 Snapshots of the configurations obtained by MC simulations of the mixed system of the $N_{t}=1296$ hard triangles and $N_{s}=648$ hard squares: (a) the initial structure, (b)-(d) the structures obtained after $10^{4}, 5 * 10^{5}$ and $8 * 10^{5}$ standard MC cycles, respectively. 
qualitative observation is presented in more quantitative way in Fig.4a-d which reveals the probability densities of finding a pair of the nearest neighbours of a given kind (SS,ST,TS,TT, where S,T denote the square and the triangle, respectively) at the distance $r^{*}=r / \sigma$, where $\sigma$ denotes the side length of the squares and the triangles.
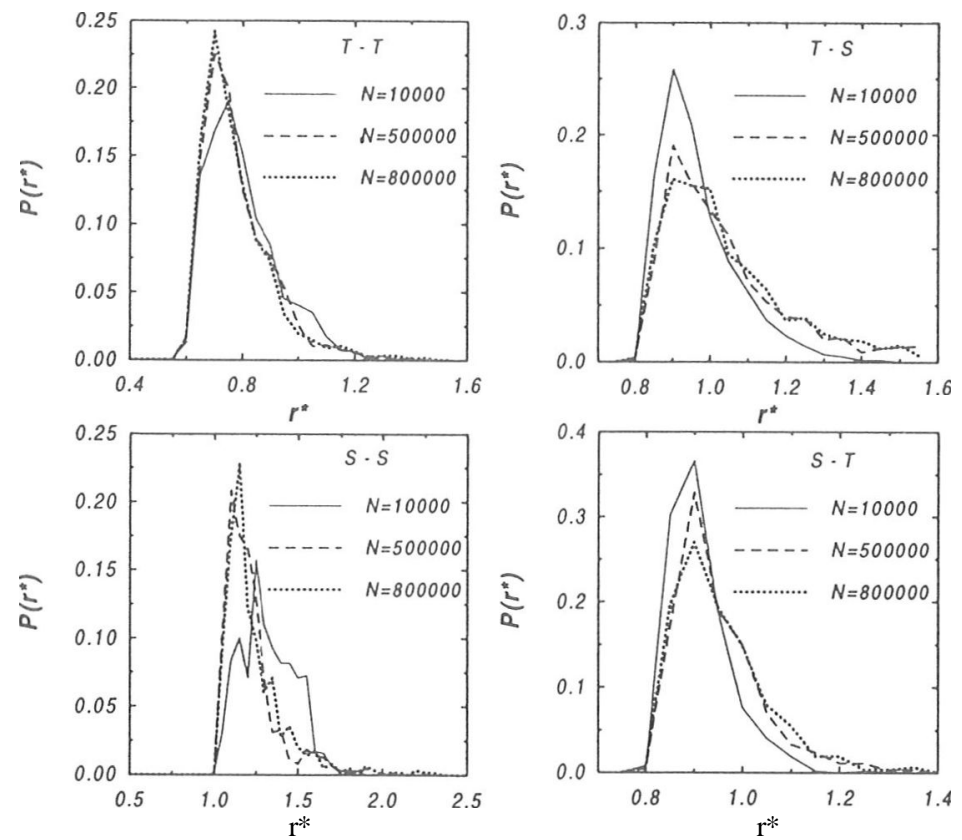

Fig.4 Probability densities of finding at the distance $r^{*}$ from the centre of a molecule $M$ the centre of the closest to it molecule $M^{\prime}$ (where $M=S, T, M^{\prime}=S, T$ ) for the structures shown in Fig.2.

The second simulation was initiated by a phase separated structure of the pure hard triangle and hard square crystals shown in Fig.5a. The simulation was performed for $N_{s}=648$ squares and $N_{t}=1440$ triangles at constant pressure equal to $\mathrm{p}^{*}=13$, but the initial relative volume was chosen as $\mathrm{v}^{*}=1.28$, i.e. by some 10 percent smaller than in the mixed case. Such a choice was taken to check if the aggregation observed in the first experiment may lead to a coexistence of the hard square solid phase and the hard triangle fluid. This is because the melting is observed near $\mathrm{p}^{*}=8$ in the pure hard square system [11], and near $\mathrm{p}^{*}=19$ in the pure hard triangle system [12], During the simulation, the relative volume increased within the first $10^{5}$ standard MC cycles, and Fig.6 suggests that it reached equilibrium at $\mathrm{v}^{*}=1.40$. This is by some four percent less than in the mixed case, what may be interpretted that the hard squares and triangles do separate indeed. In Fig.5b-d snapshots of some configurations recorded during the simulations are shown. They prove that the squares and the triangles remain separated, although some squares were solved in the fluid of the triangles. 


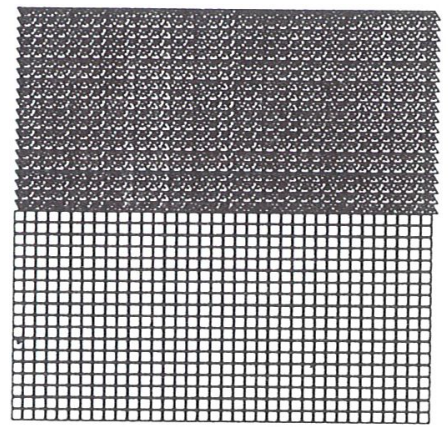

a)
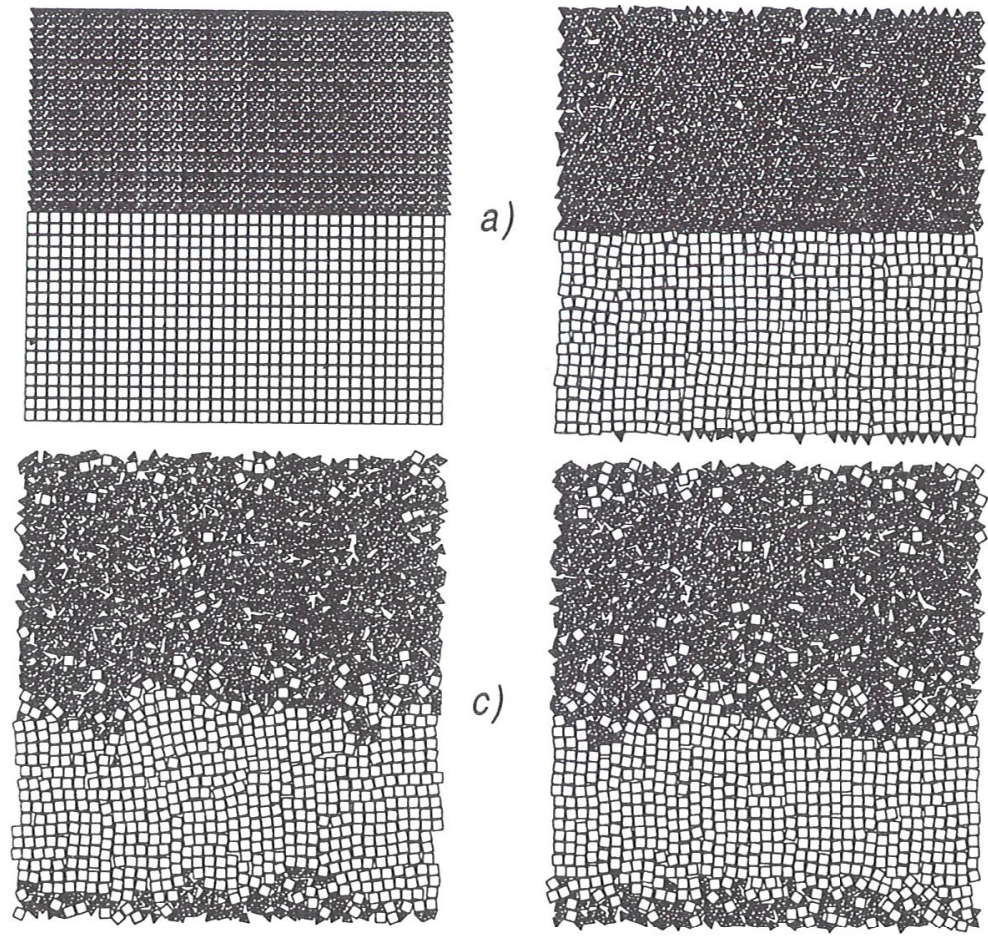

Fig.5 Snapshots of the configurations obtained by MC simulations of the phase separated system of the $N_{t}=1440$ hard triangles and $N_{s}=648$ hard squares: (a) the initial structure, (b)-(d) the structures obtained after $10^{4}, 5 * 10^{5}$ and $8 * 10^{5}$ standard MC cycles, respectively.

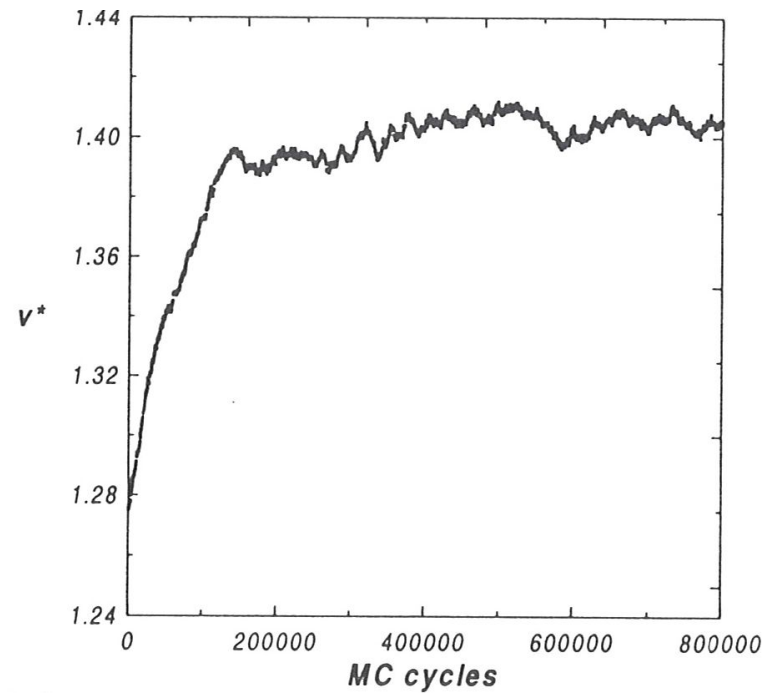

Fig.6 Relative volume as a function of the number of the standard MC cycles for the phase separated system of the $N_{t}=1440$ hard triangles and $N_{s}=648$ hard squares (see Fig.5). 
Both the slow agregation of molecules of the same kind, found in the first simulation, as well the persisting separation of different molecules, observed in the second simulation, can be thought of as arguments for phase separation in the system. However, the above experiments indicate that the time-scale neccessary to observe either a phase separation (in the first experiment) or mixing (in the second experiment) much exceeds $10^{6}$ standard MC cycles per molecule. Thus, the results described above cannot be considered as conclusive ones.

\subsection{Monte Carlo with correlated motions}

To avoid prohibitively long computations we decided to modify the probing of the configurational space of the system by introducing the correlated motions of the molecules. Their idea is presented in Fig.7. As it can be seen there, the squares were tried to be replaced by pairs of the triangles and vice versa. When a triangle was chosen randomly, one of its nearest neighbours was tossed, and next, a trial to replace such a pair (or its mirror image) by a square was undertaken in such a way that the centre of the square was equivalent to the mass centre of the pair of the triangles and the orientation of one of the diagonals of the square was equivalent to the orientation of the straight line joining the centres of the triangles (or mirror images of the triangles).
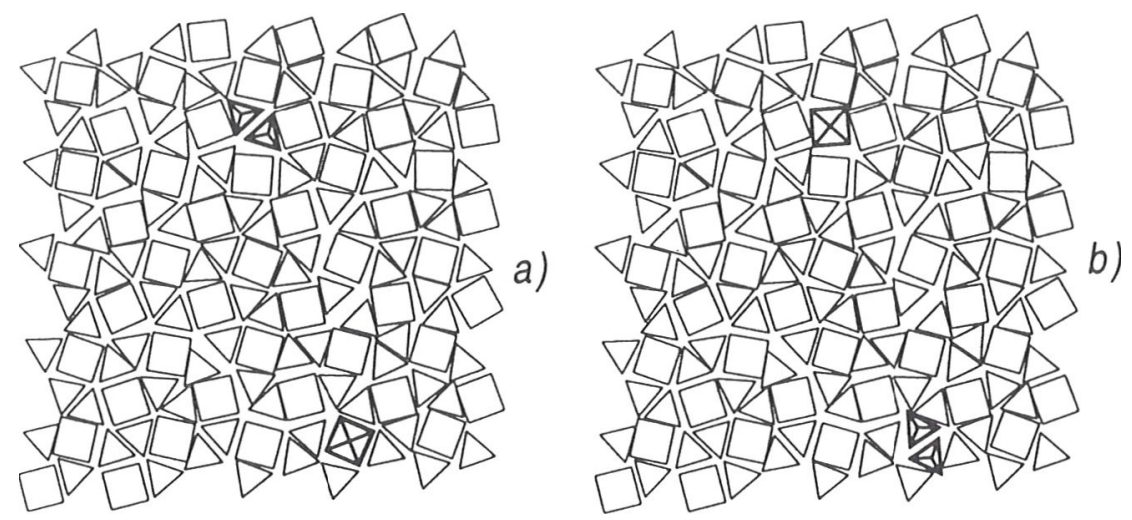

Fig.7 The example of a correlated motion. The molecules taking part in the motion are marked by line segments joining their centres with the vertices.

We started the new simulations from the final configurations obtained in the previous subsection. As it can be seen in Fig.8, within a few thousands of the modified MC cycles the structure of the phase separated system was changed drastically. The relative volume increased up to its equilibrium value, and the structure obtained after $2 * 10^{4}$ modified MC cycles containing the correlated motions was looking very similar to the fluid structure obtained in the first simulation described in section 2.1, see Fig.3d. The latter appeared to remain qualitatively 
unchanged after the same number of the modified MC cycles with correlated motions.
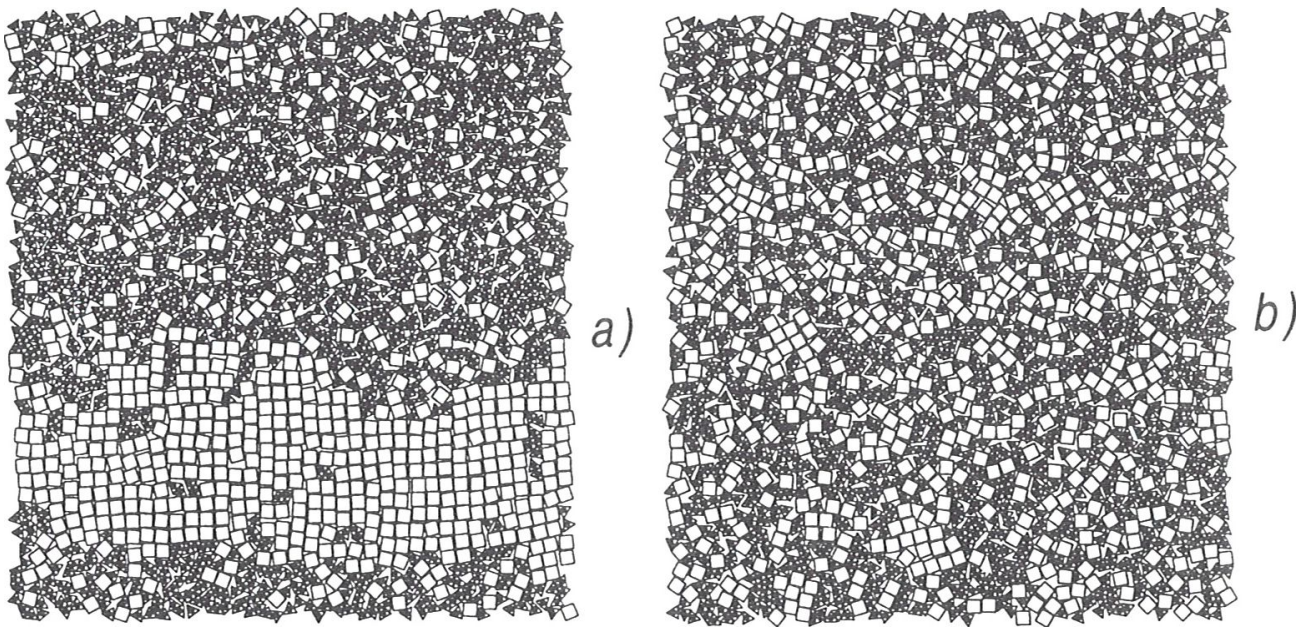

Fig.8 Snapshots of the configurations obtained by MC simulations with correlated motions starting from the structure shown in Fig.5d after: (a) $2 * 10^{3}$, and (b) $2 * 10^{4}$ modified MC cycles, respectively.

These results constitute the evidence that the hard squares and triangles do not exhibit any phase separation below the melting but form macroscopically uniform thermodynamically stable fluid phase in which some aggregation of the identical molecules can be seen at the microscopic level.

To check to what extent the above results are sensitive to any size effects, we performed test simulations with systems of linear dimensions twice as small and twice as large as the above one. We did not find any differences exceeding the experimental errors in the measured parameters of the systems studied. Hence, we expect that our conclusion concerning mixing of the squares and triangles in the fluid phase remains valid in the thermodynamic limit.

Remark'. It may be interesting to notice that molecular aggregates of the sizes similar to that observed in the system of squares and triangles slightly below the melting can be found at equilibrium in a 1:1 mixture of black and white hard discs (i.e. particles which do not differ by the size nor shape nor any other characteristic of their interaction potential) at similar relative densities, see Fig.9. This suggests strongly that these aggregates should not be considered as an indication of any phase separation but rather as a result of statistical-mechanical fluctuations. 

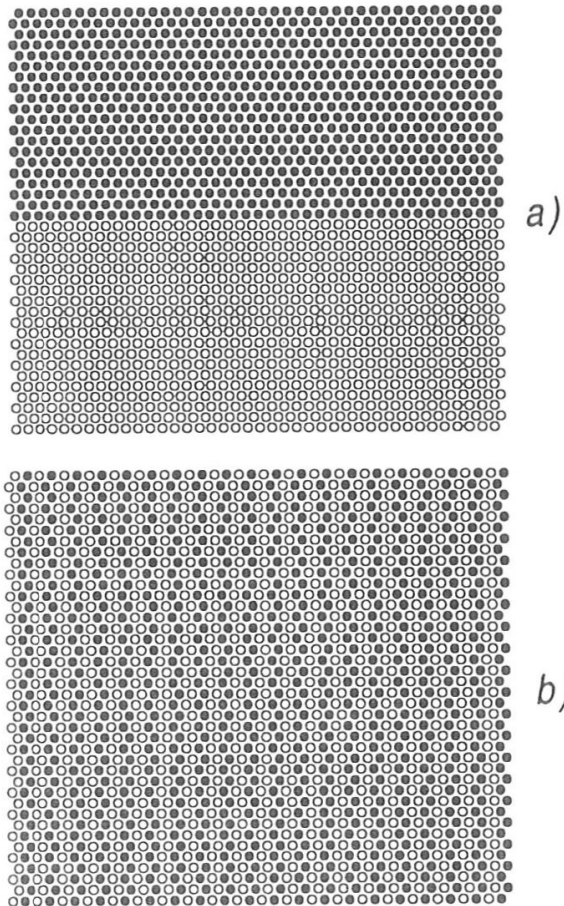

b)

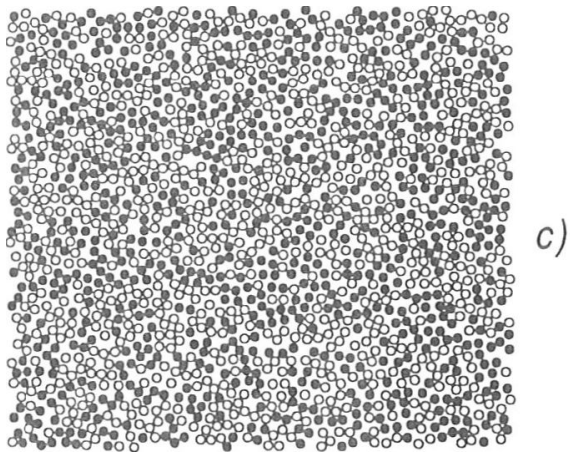

Fig.9 Snapshots of two different initial structures and the equilibrium structure of a system of $N_{b}=N_{w}=800$ black and white hard discs of the same diameter.

\section{Summary and conclusions}

Using the MC simulations we studied the continuous model of the hard squares and triangles of equal sides at densities slightly below the spontaneous melting of the system. The question we posed concerned the existence of a phase separation below the melting in such a system. We found that the standard 
simulations, using diffusive motions of the molecules, are inconclusive in answering this question. By introducing the correlated motions which much increase the efficiency of the Monte Carlo simulations (in the present case the equilibration time was reduced by about two orders of magnitude) we succeeded to show, without performing thermodynamic integration of any equations of state, that squares and triangles do not separate below melting.

It is worth to add that at the microscopic level some spontaneous aggregation of identical molecules was observed in the hard square-triangle system close to melting. In experiments performed with a 1:1 dense binary mixture of black and white hard discs, with differ by no feature except the color, similar aggregation clearly caused by thermodynamic fluctuations - was found at equilibrium. This indicates that the microscopic aggregation found in the square-triangle system should not be considered as a precoursor of any phase separation but as an effect of thermodynamic fluctuations.

\section{Acknowledgments}

This work was supported by the Committee for Scientific Research (KBN) within the grant $8 \mathrm{~T} 11 \mathrm{~F} 01008 \mathrm{p} 04$. Computer simulations were performed at Poznań Computer and Networking Centre (PCSS). We are grateful to Professor Jerzy Małecki for helpful remarks and to Dr. Arkadiusz Brańka for reading the manuscript.

\section{References}

[1] T. Biben and J.-P. Hansen, Phys. Rev. Lett. 66, 2215 (1991).

[2] R. van Roij and B. Mulder, J. Phys. II France 4, 1763 (1994).

[3] S. Asakura and F. Oosawa, J. Chem. Phys. 22, 1255 (1954).

[4] W. G. Hoover, W. T. Ashurst, and R. Grover, J. Chem. Phys. 57, 1259 (1972);

W. G. Hoover, N. E. Hoover, and K. Henson, J. Chem. Phys. 70, 1837 (1979).

[5] K. W. Wojciechowski, Physica A232, 723 (1996).

[6] R. Collins, Proc. Phys. Soc. 83, 553 (1964). Generalization of the model proposed by Collins is discussed by M. A. Glaser and N. A. Clark, Adv. Chem. Phys. Vol. LXXXIII, 543 (1993).

[7] H. Kawamura, Prog. Theor. Phys. 61, 1584 (1979).

[8] H. Kawamura, Prog. Theor. Phys. 70, 352 (1983); Physica A177, 73 (1991).

[9] M. Widom, Phys. Rev. Lett. 70, 2094 (1993).

[10] P. A. Kalugin, J. Phys. A27, 3599 (1994).

[11] K. W. Wojciechowski and D. Frenkel, unpublished.

[12] K. W. Wojciechowski, unpublished. 